\title{
Artificial Intelligence in Assisted Reproductive Technology: Present and Future
}

\author{
Rutvij J Dalal ${ }^{1}$, Sahil Gupta ${ }^{2}$, Akanksha P Mishra ${ }^{3}$
}

\begin{abstract}
Artificial intelligence (Al) has found its way into medicine in the form of robotics, operational and computational tools. We have software to store and recall a patient's history instantly and algorithms to decide the course of treatment depending on past data. We have robots performing surgeries and witnessing systems to help prevent human errors. There have been significant advancements in the incorporation of Al in the artificial reproduction technology (ART) labs. In vitro fertilization (IVF) at present is a very subjective science, depending on the expertise and experience of the operators, mainly embryologists. Automation and Al are expected to bring about a more calculated, computed, and standardized approach to IVF. Presently, Al is used in the IVF lab for witnessing, data collection, record maintenance, and selecting the best possible embryo for transfer. Continuous research is being undertaken towards bringing more and more automation in the form of robotics. Artificial intelligence in ART is a very exciting upcoming field of research. Our review enlists the present Al in an ART lab and its future prospects. Keywords: Artificial intelligence, Artificial reproduction technology, Assisted reproductive technique.

International Journal of Infertility and Fetal Medicine (2020): 10.5005/jp-journals-10016-1208
\end{abstract}

\section{INTRODUCTION}

Today the world of in vitro fertilization (IVF) and reproductive medicine, in general, has witnessed a phenomenal surge in newer technologies including, but not limited to, additions like preimplantation genetic screening, endometrial receptivity assay, time-lapse monitoring of embryos, oocyte vitrification, laser hatching, and advancements in culturing systems. Time-lapse monitoring can be considered to be a "semi-automated" system wherein complex algorithms would assist in better embryo selection for transfer. Still, challenges remain. For example, even today live birth rates after one attempt at embryo transfer hovers below 40\% (according to ESHRE). Artificial intelligence (Al) aims to bring a revolutionary paradigm shift in artificial reproduction technology (ART) success rates.

Although the first attempts at using Al date back to as early as the 1990s, its application in improving ART success rates is fairly recent. To understand how AI can influence IVF results or management, a brief working knowledge of how Al works is useful. Other branches of medicine have seen a significant penetration and benefit from the application of Al technologies in clinical practice. One reason why it is convenient today to incorporate Al is the sophisticated computing tools not available in the past. What complements the powerful computers is the ever-growing data collected and analyzed over hundreds of thousands of cases. With a massive amount of data and computing, today it is possible to design complex algorithms that are not only useful in management but also backed by evidence.

The obvious question at this point is-will Al eventually take over human systems in an IVF lab setting? Will the decisionmaking by expert clinicians and experienced embryologists be deemed redundant by an artificial computer? Well, even though it is too early to answer that question right now it seems to be a remote possibility. Artificial intelligence, as of today, seems to be a powerful tool that can complement and assist professionals in their task of handling infertility, not a replacement. Just like systematic reviews and meta-analyses that aim to incorporate tons of data and
1Department of IVF, Janini IVF, New Delhi, India

${ }^{2}$ Department of IVF, Aveya Fertility, New Delhi, India

${ }^{3}$ Department of IVF, Lilavati Hospital and Research Center, Mumbai, Maharashtra, India

Corresponding Author: Rutvij J Dalal, Department of IVF, Janini IVF, New Delhi, India, Phone: +91 9930273377, e-mail: rutvij.dalal@gmail. com

How to cite this article: Dalal RJ, Gupta S, Mishra AP. Artificial Intelligence in Assisted Reproductive Technology: Present and Future. Int J Infertil Fetal Med 2020;11(3):61-64.

Source of support: Nil

Conflict of interest: None

conclude meaningful deductions, Al can analyze gigabytes of data across the globe and demise useful pragmatic algorithms for ART management-for clinicians and embryologists alike.

The core areas where $\mathrm{Al}$ seems to be of utmost relevance include sperm selection during intracytoplasmic sperm injection (ICSI), assessing ovarian reserve, embryo selection for transfer and emerging new treatments (adjuvants) in IVF, etc. Artificial intelligence has tremendous potential to be of assistance in areas like data storage and processing, data processing including audits, quality control/quality assessment analytics, and witnessing system to avoid human errors in the embryology lab. Artificial intelligence can also be put to much clinical use by assessing couples' need for egg donors or sperm donors and matching them appropriately with available donors utilizing tools like facial symmetry. Artificial intelligence is already being used in the IVF setup presently in three major formats: (1) natural language processing (NLP), (2) machine learning, and (3) robotics. ${ }^{1}$

Natural language processing involves finding the useful "sound" out of white noise-extracting relevant, useful conjectures out of jargons of data that are piled up out of thousands of cycles, e.g., electronic medical records. Natural language processing organizes chaotic data and thereby assists the machine learning tool. Machine learning builds a mathematical model out of "training 
data" to make decisions or for prognostication. It consists of three parts: (i) unsupervised learning, (ii) supervised learning, and (iii) reinforcement learning.

\section{Artificial Intelligence in the Present ART Practice}

Artificial intelligence has found its way into ART in the form of algorithms predicting the gamete/embryo quality based on a certain set of data. Currently, the assessment is done manually by embryologists, inserting the possibility of human error and bias. Where humans are involved, any assessment is subjective depending on the operator's experience and personal conclusions. Also, these assessments are based on morphology alone.

The most important predictor of pregnancy is oocyte quality. At present, there is no noninvasive method to determine aneuploidy in oocytes, since aneuploid oocytes are mostly, no different morphologically from the normal ones. Currently, oocytes are assessed based on their maturity, cytoplasmic inclusions, the perivitelline space, polar body, and homo/heterogeneity of the cytoplasm.

With the application of $\mathrm{Al}$, it might be possible to develop an algorithm to determine the developmental competence of an oocyte. The presently available methods that may be customized for this purpose are time-lapse, metabolomics, or transcriptomics. Polarized microscopy can non-invasively detect spindle abnormalities in the oocytes. ${ }^{2}$ An oocyte may have extruded the polar body, but if it persists in the telophase I stage, it has a low developmental competence. Similarly, time-lapse bright-field microscopy coupled with particle image velocimetry provides an image analysis of the transition of the oocyte from germinal vesicle to metaphase II stage, providing valuable information regarding the developmental potential of the oocytes. ${ }^{3}$

Similar to the oocytes, embryos are commonly graded by embryologists based on their morphology. Selecting the most viable embryo from the cohort is critical when the aim is to transfer a single embryo to avoid multiple pregnancies. One convenient way to weed out the nonviable embryos is to extend their culture to blastocysts. This provides the first line of selection to choose a viable embryo to transfer. However, this is not to say that all blastocysts are euploid and have similar implantation potential. They are in turn graded based on their expansion, trophectoderm, and inner cell mass. Blastocysts grading has proven to be highly subjective and prone to inter- and intraobserver variation. Even the exact evaluation time chosen for grading makes a difference to the grading as blastocyst is a dynamic entity. Automation of the grading system by use of computer imaging and precise algorithms can eliminate inter- and intraobserver variation. Computer imaging by time-lapse monitoring coupled with a modern machine learning algorithm can also take into account the morphokinetic events in determining the viability of an embryo. ${ }^{4}$

Artificial intelligence was recently applied to PGT-A (Cooper Surgical PGTai) in the form of a machine learning approach, to eliminate operator subjectivity, for reporting and interpreting next-generation sequencing results. ${ }^{5}$

Artificial intelligence-based programs like computer-assisted semen analysis (CASA) exist to assess sperm morphology and kinetics subjectively. However, it is riddled with some problems like its inability to distinguish between debris and dead sperm. ${ }^{6}$ It has also been stated that the CASA program perhaps needs further improvement before it can be confidently put to mainstream clinical use. ${ }^{7}$
Robotic surgery has been used in reproductive surgeries like tubal reanastomosis, myomectomy, endometriosis surgery, and ovarian transplantation. ${ }^{8}$ Presently, robotic surgery poses certain problems like cost, bulky equipment, lack of tactile feedback, and locking of patient position, scientists around the world are working to improve the precision and ergonomics of existing systems.

\section{Future of Artificial Intelligence in Artificial Reproduction Technology}

The crucial aspects of creating a viable embryo in the lab are selecting the best sperm to fertilize the oocytes, oocyte grading, embryo culture and grading, blastocysts grading; and mapping the journey of the oocyte to blastocyst to assess the potential of a particular embryo based on the time taken to achieve its milestone. There are various individual Al programs already available to facilitate every step of the embryo culture. Is it then possible to entirely replace the skilled embryologist with Al? It would seem so.

\section{Reproductive Surgeries}

Say a couple walks into an IVF facility with the diagnosis of fibroids, or endometriosis interfering with their fertility. They can be offered a minimally invasive robotics surgery to overcome the problem.

\section{Hormone Analysis}

Most of the pathology labs are automated, so we have the hormone profile generated with the help of Al.

\section{Ovarian Stimulation Protocol}

A 2018 literature review conclusively proved the relationship between serum AMH levels and the ovary's response to stimulation. ${ }^{9}$ The main criteria in determining a stimulation protocol for a particular patient are age, $\mathrm{AMH}, \mathrm{BMI}$, and previous response to stimulation (if applicable). The software can be developed with this nomogram, considering all the determining aspects of the woman's ovarian status to suggest the most suitable protocol to stimulate the patient's ovaries.

\section{Evaluation of the Oocytes}

The oocyte quality plays a pivotal role in the success of an ART cycle. The perfect way to determine the quality of an oocyte will be inexpensive, non-invasive, and unobtrusive. Morphological evaluation of oocytes is of little help in assessing their potential. By taking pictures of the oocyte from the germinal vesicle to the metaphase II stage via time-lapse imaging, it is possible to analyze its cytoplasmic movement velocity (CMV) profile using the particle image velocimetry method to identify the competency of mouse oocytes with an accuracy of over $91 \% .{ }^{10}$ Furthermore, Yanez et al. ${ }^{11}$ discovered that in the hours after fertilization, nondestructive measurement of the viscoelastic property of the zygote could predict blastocysts formation with $>90 \%$ precision. Moreover, they concluded that a transcriptomic evaluation of the developing embryos can give a very fair assessment of viability. A collective application of the above techniques may assist in singling out the best oocyte among the cohort.

\section{Sperm Selection}

Selecting a morphologically and genetically normal sperm is as crucial as a normal oocyte for viable embryo generation. Currently, the Al system used in semen analysis is CASA, but it is not without its setbacks. In 2014, Sahoo and $\mathrm{Kumar}^{12}$ used five Al techniques 
and applied eight feature selection methods to find out the main attributes predicting the fertility rate in human males.

\section{Intracytoplasmic Sperm Injection}

Proceeding to ICSI, software like IMSI and MSOME are already available to select the most morphologically normal sperm. The argument being that normal sperm nucleus positively correlated with ICSI fertilization rate and pregnancy outcome. ${ }^{13}$ Zhang et al..$^{14}$ claim to have developed a robotic system for tracking (with $96 \%$ accuracy) and immobilizing the target sperm with $94.5 \%$ success regardless of the sperm's velocity or orientation. Lu et al. in 2011 came up with a robotic ICSI machine with no human input other than a few clicks of the mouse, and with a success rate of $90 \%{ }^{15}$

\section{Embryo Culture and Tracking}

Embryos are traditionally cultured in static medium in a Petri dish. However, these are not natural cultural conditions. In vivo, the embryo is continuously exposed to changing medium composition along the length of the fallopian tube, and metabolites like ammonia are constantly removed. ${ }^{16}$ Meseguer et al. ${ }^{17}$ have proposed a dynamic model of a microfluidics system that continuously changes the culture medium surrounding the developing embryo. This, if coupled with a time-lapse imaging system may provide the ideal culture environment with the ideal tool for assessment of the embryo development and milestone. The current time-lapse program, with the help of a series of algorithms based on morphokinetic parameters and embryo morphology, can predict the embryos with the lowest implantation potential. ${ }^{18}$

\section{Embryo Freezing}

Automated devices are available to assist embryo vitrification. They claim to be less labor and skill intensive, at the same time reducing interoperator variance. ${ }^{19}$

\section{Conclusion}

There is a huge prospect for the integration of Al in the ART lab. From hormone assessment to oocyte grading, sperm selection, ICSI, denuding, embryo culture, embryo development tracking and assessment to vitrification, the advantages of Al may be manifold in performing tedious and repetitive tasks with high reproducibility, low error, record maintenance, and mechanical judgment. The challenges may be the cost and the ethical consideration of allocating the job of human decision-making to a computer. A machine may never be able to replace the human touch and compassion.

In this paper, we have enlisted the current and proposed Al programs that are or can be integrated into the IVF lab for automation. In the future, the combined knowledge of these $\mathrm{Al}$ tools might have the potential to create and select the best embryo for implantation and bring about standardization in the pregnancy rates all over the world, independent of the human component. Over time these techniques are likely to improve. With more cases and data mining, these programs will self-learn to get better with time.

There is a possibility that with the advent of automation in ART, a lot of embryologists may lose their jobs. Or worse, their skill. They may get relegated to simply assisting the machines in performing the actual embryology. A lot of guidelines will have to be laid to make the transition from human to robot as ethical as possible.
No doubt technology will only improve but we need to address serious questions.

Are we going to rely entirely on the software for embryo quality assessment, or will the embryologist be the final decision maker? Is there any clear and present advantage of having a fully automated laboratory? To what extent will man or machine be allowed to make final choices? Perhaps we will have to learn to coexist with the machines and make them work for us. The amount of responsibility that can be allocated to the machines has to be decided by us, over time, and help us shape the role of the embryologist inside an ART lab.

\section{References}

1. Wang $R$, Pan $W$, Jin $L$, et al. Artificial intelligence in reproductive medicine. Reproduction 2019;158(4):R139-R154. DOI: 10.1530/REP18-0523.

2. Keefe $\mathrm{D}$, Kumar M, Kalmbach K. Oocyte competency is the key to embryo potential. Fertil Steril 2015;103(2):317-322. DOI: 10.1016/j. fertnstert.2014.12.115.

3. Hien Bui TT, Belli M, Fassina L, et al. Cytoplasmic movement profiles of mouse surrounding nucleolus and not-surrounding nucleolus antral oocytes during meiotic resumption. Mol Reprod Dev 2017;84(5):356362. DOI: $10.1002 / \mathrm{mrd} .22788$

4. Kragh MF, Rimestad J, Berntsen J, et al. Automatic grading of human blastocysts from time-lapse imaging. Comput Biol Med 2019;115:103494. DOI: 10.1016/j.compbiomed.2019.103494.

5. Zaninovic N, Elemento O, Rosenwaks Z. Artificial intelligence: its applications in reproductive medicine and the assisted reproductive technologies. Fertil Steril 2019;112(1):28-30. DOI: 10.1016/j. fertnstert.2019.05.019.

6. Larsen L, Scheike T, Jensen TK, et al. The Danish first pregnancy planner study team. Computer-assisted semen analysis parameters as predictors for fertility of men from the general population. Hum Reprod 2000;15(7):1562-1567. DOI: 10.1093/humrep/15.7.1562.

7. Talarczyk-Desole J, Berger A, Taszarek-Hauke G, et al. Manual vs. computer-assisted sperm analysis: can CASA replace manual assessment of human semen in clinical practice? Ginekol Pol 2017;88(2):56-60. DOI: 10.5603/GP.a2017.0012.

8. Taylan E, Oktay. KH, Robotics in reproduction, fertility preservation, and ovarian transplantation. Published online 2017 Feb 27.

9. Iwase A, Osuka S, Goto M, et al. Clinical application of serum antiMüllerian hormone as an ovarian reserve marker: a review of recent studies. J Obstet Gynaecol Res 2018;44(6):998-1006. DOI: 10.1111/ jog.13633.

10. Cavalera F, Zanoni M, Merico V, et al. A neural network-based identification of developmentally competent or incompetent mouse fully-grown oocytes. J Vis Exp 2018(133):56668. DOI: 10.3791/56668.

11. Yanez LZ, Han J, Behr BB, et al. Human oocyte developmental potential is predicted by mechanical properties within hours after fertilization. Nat Commun 2016;7(1):10809. DOI: 10.1038/ ncomms10809.

12. Sahoo AJ, Kumar Y. Seminal quality prediction using data mining methods. Technol Health Care 2014;22(4):531-545. DOI: 10.3233/ THC-140816.

13. Bartoov B, Berkovitz A, Eltes F, et al. Real-time fine morphology of motile human sperm cells is associated with IVF-ICSI outcome. J Androl 2002;23(1):1-8. DOI: 10.1002/j.1939-4640.2002.tb02595.x.

14. Zhang Z, Dai C, Huang J, et al. Robotic immobilization of motile sperm for clinical intracytoplasmic sperm injection. IEEE Trans Biomed Eng 2019;66(2):444-452. DOI: 10.1109/TBME.2018.2848972.

15. Lu Z, Zhang X, Leung C, et al. Robotic ICSI (intracytoplasmic sperm injection). IEEE Trans Biomed Eng 2011;58(7):2102-2108. DOI: 10.1109/ TBME.2011.2146781.

16. Rejniak KA, Kliman HJ, Fauci LJ. A computational model of the mechanics of growth of the villous trophoblast bilayer. Bull Math Biol 2004;66(2):199-232. DOI: 10.1016/j.bulm.2003.06.001. 
17. Meseguer $M$, Kruhne $U$, Laursen S. Full in vitro fertilization laboratory mechanization: toward robotic assisted reproduction? Fertil Steril 2012;97(6):1277-1286. DOI: 10.1016/j.fertnstert.2012.03.013.

18. Carrasco B, Arroyo G, Gil Y, et al. Selecting embryos with the highest implantation potential using data mining and decision tree based on classical embryo morphology and morphokinetics. J Assist Reprod Genet 2017;34(8):983-990. DOI: 10.1007/s10815-0170955-x.

19. Roy TK, Brandi S, Peura TT. Chapter 20 gavi-automated vitrification instrument. Methods Mol Biol 2017;1568:261-277. 